\title{
Production of nanoparticles, of powders and setup of components for power equipment
}

\author{
E A Patroi1, A Bordianu2 and G Samoilescu3 \\ ${ }^{1}$ INCDIE ICPE-CA- Bucharest, Romania \\ ${ }^{2}$ Electrical Engineering Faculty, University Polytechnics Bucharest, Romania \\ 3 „Mircea cel Bătrân” Naval Academy , Constanţa, Romania \\ samoilescugheorghe@yahoo.com
}

\begin{abstract}
The paper presents ways to produce magnetic nanoparticles to be incorporated into magneto-bioelectronic devices where chemical processing must control composition, microstructure, phase purity, particle morphology, and control size particle, thereby reducing particle aggregation and size distribution. The methods of synthesis of magnetic nanoparticles are analyzed: physical vapor deposition, mechanical and chemical synthesis in the solution. Solution synthesis offers advantages: easy control of the size and composition of the particles, possibilities to change the surface in order to obtain a stable dispersion in different solid or liquid media. The properties of nanoscale powders, nanostructured materials and nanoparticles in solution are strongly dependent on particle size, and their dispersion state. Laboratory syntheses have been performed by analyzing the methods of synthesis of nanometric magnetic particles.
\end{abstract}

Keywords: magnetic nanoparticles, magneto-electronic device, chemical processing, nanoscale powders, nanostructured materials

\section{Introduction}

Magnetic nanoparticles are inorganic materials and should be obtained with controlled properties (by manipulating processing parameters). For a safe reproducibility it is essential to know the most sensitive synthesis parameters with a view to obtaining the particles in the desired process step. It is also necessary to control the processing conditions in order to manipulate the structural characteristics of the particles, which will allow control of the intrinsic magnetic properties [1,2].

The most common methods of synthesis of magnetic nanoparticles are: physical vapor deposition, mechanical (grinding) [3,4] and chemical synthesis in solution [5-8]. Both in the vapor phase and in solution, the particles are composed of individual atoms. Mechanical nanostructures can be obtained by grinding some alloys (in bulk). Chemical methods for the production of magnetic nanoparticles are preferred because of their increased molecular homogeneity, production cost efficiency, particle size control and particle size distribution, morphology and conglomerate size.

For magnetic applications, composite materials with polymeric matrix and magnetic inclusions in the form of powder (ferrite, iron, nickel, etc.) are most often used. The result is a macroscopically homogeneous material with high performance compared to traditional materials. The use of composite materials with polymeric matrices brings many advantages, for example a thermosetting matrix composite material and graphite inclusions which may be much stronger than steel.

Turkevich was the one who established the first standard reproducible procedure for the preparation of metallic colloids [9] and proposed a mechanism for the stepwise formation of nanoclusters based on nucleation, growth and agglomeration $[10,11]$. His model was later modified 
due to the data provided by modern analytical techniques and the results obtained from thermodynamic and kinetic studies. According to this model, the metal salt is first reduced to give metallic zero-valent metal atoms colliding in the solution to obtain an incipient stable nucleus whose formation is irreversible. It is assumed that in order to initiate nucleation, the concentration of the metal atoms in the solution must be large enough to reach a specific concentration called "supersaturation" $[12,13]$.

LaMer [14] proposed the idea that nucleation from supersaturated solutions appears explosive. At the critical point of supersaturation, stable nuclei begin to grow. Growth follows the Ostwald kinetics of diffusion and maturation (formation of large nuclei at the expense of the smallest) [15]. According to this model, monodispersion is a consequence of the careful separation of the two steps: the nuclear phase and the subsequent growth stage. For the metallic particles, the nucleation is the result of complex interactions between factors such as the reaction conditions and the difference between the reduction potential of the metal salt and reducing agent. Reaction conditions include the rate of addition, the rate of removal, the reaction temperature, the reaction time, and even the agitation rate.

To obtain a monodisperse sample, the nucleation step must be complete before the growth stage begins. As a rule, achieving short nuclear nucleation is a prerequisite for the formation of monodispersed particles $[16,17]$.

As a result of the van der Waals forces and the system's tendency to minimize total surface or interfacial energy, nanostructured particles agglomerate. This can happen during synthesis (during the drying process), handling and/ or post-processing. To prevent agglomeration of particles, surfactants (any substance that influence the surface tension or interfacial tension of the environment in which it is dissolved) [18] are used to control dispersion during chemical synthesis by reducing particle interactions (through an increase in repulsion forces). Stabilizers can also be used to control particle size and shape.

Sol-gel and hydrothermal reactions lead to the production of oxide nanoparticles. This is due to the fact that the synthesis medium is an aqueous solution without a source of reduction. For the realization of the metal nanoparticles reactions are used which must take place under very mild conditions (low temperatures), avoiding the formation of mixed oxide systems and for which a rigorous control of the surface can be achieved.

One of the techniques used for particle synthesis is the precipitation of the products from the solution. From the kinetic point of view, the precursors are dissolved in a solvent and a precipitating agent which is added to form insoluble particles. Many magnetic nanoparticles can be synthesized using these precipitation reactions in aqueous solutions. These reactions can generate a wide range of magnetic materials, including spinel or perovskite, metal and metal alloys respectively. Precipitation allows for large amounts of particles, but the size distribution is usually high.

Reduction of transition metal salts is easier and most commonly used for the preparation of metal nanoparticles. Reducing agents are also borohydride derivatives. This method provides a simple synthesis pathway for $\mathrm{Fe}$, $\mathrm{Co}$ and $\mathrm{Ni}$ nanoparticles as well as for the $\mathrm{Fe} / \mathrm{Pd}$ alloy but has the disadvantage of embedding the boron in the particles that undergo a change in magnetic properties. For example, Co particles were obtained by the microemulsion method in a binary system, such as DDAB (didodecyldimethylammonium bromide)/ in toluene by reducing $\mathrm{CoCl} 2$ with $\mathrm{NaBH} 4$. The average particle size of the prepared samples can be changed from 1.8-4.4 $\mathrm{nm}$ by controlling the $\mathrm{CoCl} 2$ concentration in the DDAB solution in toluene. TEM studies have shown that these particles are uniform and well-isolated.

The coercivity of the particles at $10^{\circ} \mathrm{K}$ increases from 640 to 1250 Oe whereas the particle size increases from 1.8 to $4.4 \mathrm{~nm}$ and the particle blocking temperature increases from 19 to $50^{\circ} \mathrm{K}$ for the same grain range. Saturation magnification at $20 \mathrm{~K}$ increases with decreasing particle size and reaches a value of about $200 \mathrm{emu} / \mathrm{g}$ with $20 \%$ greater than the value obtained in the crystalline mass for particles with an average size of $1.8 \mathrm{~nm}$, thus implying an increase in the moment magnetic on the atom in the nanoparticle system. Increasing the temperature has led to the formation of clusters with a higher blocking temperature. 
A similar method can be used to prepare oxidized Fe particles by passivation with a fluoride layer. $\mathrm{Fe} / \mathrm{Co}$ and $\mathrm{Fe} / \mathrm{Pd}$ alloys were obtained.

Bonnemann [19] developed the reduction method, derived from the previous process, but involving both reducing and stabilizing agents for particle growth (alkyl ammonium borohydrides).

Pileni synthesized cobalt nanoparticles by reducing $\mathrm{CoCl} 2$ with $\mathrm{NaBH} 4$ in the presence of [bis (2ethylhexyl) sulfosuccinate]] sodium [20]. The particles were spontaneously assembled into planar superstructures and could be processed in spatial over-networks. Decomposition reactions (thermolysis) at low temperatures can be made from organometallic compound type precursors [21].

Among these, very good results can be achieved with carbonyl metal or metal-olefinic compounds containing an olefinic or polyolefinic ligand capable of being hydrogenated to give a single metal atom that might condense in the reaction medium.

Metal carbonyls are readily obtainable precursors, used for a long time for the synthesis of Co and Fe magnetic colloids.

The discovery of metal-carbonyl complexes by thermolysis leads to the production of nanopowders, nanostructured materials and nanoparticles in solution, the size and shape of which can be controlled by the reaction medium.

This method involves reactions in organic solutions containing metal carbonyls: $\mathrm{Fe}, \mathrm{Co}, \mathrm{Ni}, \mathrm{Pt}$, compounds that decompose at low temperatures. The chemical reduction to metal is complete after a strong stirring of the solution. The colloidal solution formed precipitates upon cooling.

The parameters that influence the process are: decomposition temperature; solution dilution; the precipitating reagent used; precipitation temperature; present in systems considered one or two metals; the presence of surfactants or ligands.

The thermolysis of $\mathrm{Co} 2$ (CO) 8 in solution, in the presence of trioctylphosphine oxide (TOPO) leads to the production of cobalt nanoparticles. High temperature rapid thermolysis (1810C) of the same precursor in the presence of organic molecules containing long chain alkyl chains and capable of serving as surfactants or ligands (oleic acid, triacyl phosphoric acid or TOPO triacylphosphine oxide) allows the production of spherical monodisperse nanocrystals in the $3-17 \mathrm{~nm}$. The decomposition of $\mathrm{Fe}$ (CO) 5 by thermolysis leads to the production of metallic nanoparticles whose size and shape can be controlled by the reaction medium. This process has been recently extended to the preparation of bi- and even trimetallic nanoparticles.

Metal-olefin compounds, by mild decomposition, into molecular hydrogen, lead to metallic nanoparticles. Thus, $\mathrm{Co}(\mathrm{C} 8 \mathrm{H} 13)(\mathrm{C} 8 \mathrm{H} 12)$ rapidly decomposes at room temperature in low pressure solution ( $3 \mathrm{bar}$ ) in the presence of molecular hydrogen to produce cobalt nanoparticles $(4 \mathrm{~nm})$. Nickel nanoparticles can be prepared in the same kind of Ni (C8H12) 2. The particles have a size of 4-5 nm and adopt the cubic structure with centered faces of the nickel crystal.

The method of chemical vapor deposition of organometallic compounds (MOCVD) is a cheap and versatile method, operating without expensive vacuum equipment compared to physical methods. Conventional techniques of the MOCVD method require molecules with volatile and constant vapor pressure [22].

Volatile precursors with sufficient thermal stability during the evaporation process are required to function as a messenger for the material to be deposited. Being unreactive with anything else in the vapor phase, this is a necessary supplement for multimetal materials.

Precursors for CVD are typically metal alkoxides, $M$ (OR) n, $\beta$-diketones, alkyl or cyclopentadienyl derivatives, volatile hydroxyamines. Molecular chemistry offers great flexibility in choosing ligands to meet the requirements of MOCVD precursors.

The microemulsion technique consists in obtaining a microemulsion of water in a non-polar medium (e.g., petroleum ether) using a non-ionic surfactant (e.g., polyoxyethylene-nonyl phenol ether). Despite the fact that the reaction speed is very high, no particle agglomeration occurs (if agitation continues for several hours flocculation phenomena occur but the introduction in an ultrasonic bath allows reduction to the original size). 
Emulsions are dispersed systems consisting of two liquids insoluble to one another, one of which is in a state of advanced dispersion. Dispersion of a liquid into the mass of another liquid can be accomplished by mechanical mixing. A liquid-liquid dispersion system can not be referred to as an emulsion unless it has a certain solubility. At emulsification, the mechanical work spent leads to the superficial energy increase of the system. The larger the stored energy, the more unstable the emulsion tends to reduce the surface of the system by joining the droplets when they come in contact due to the Brownian motion. The reduction of collision probability can be achieved by decreasing the kinetic energy, respectively by increasing the viscosity / decrease of the temperature and the electrostatic repulsion of the droplets, respectively, due to the adsorption of polar substances or ions. The only possibility is the decrease of the interfacial tension c, which is achieved by means of emulsifiers. The most used emulsifiers are surfactants, which decrease the value of the interfacial tension of the liquids in contact [23]. The method in which a polyol acts as a solvent, reducing agent and surfactant is a suitable method for obtaining nanoscale particles of controlled shape and size [24]. Particle inclusions range in size from a few nanometers to a few millimeters. Their shape may be spherical, cubic, flat, regular or irregular geometry [25], etc. In most composites, particle orientation is random.

The fibers have rays from several nanometers to 5 and $15 \mu \mathrm{m}$. They may be short (they are also called discontinuous because the general appearance in the array is discontinuous), long (or continuous), unidirectional, multidimensional, etc. Fiber yields composite material with mechanical strength, hardness, lightness, and the arrangement of the material requires anisotropy of the material. Fiber generally occupies between $30 \%$ and $70 \%$ of the total volume of a composite material [25-28].

\section{Synthesis of Fe nanoparticles and Fe-Co alloy}

Fe nanoparticles were obtained in the system: FeCl3.6H2O - NaBH4 - PEG

For synthesis, the following raw materials were used: $\mathrm{FeCl} 3 \cdot 6 \mathrm{H} 2 \mathrm{O}$ hexahydrate chloride of $98 \%$ purity, FLUKA origin; 99\% NaBH4 sodium borohydride, Aldrich origin; Ethyl alcohol p.a. of the Chemical Company; PolyethyleneglycolPEG of Fluka origin

The preparation of $\mathrm{Fe}$ nanoparticles was accomplished by reducing iron chloride hexahydrate $\mathrm{FeCl} 3.6 \mathrm{H} 2 \mathrm{O}$ and direct precipitation of $\mathrm{Fe}$ nanoparticles from an aqueous solution containing a sodium borohydride NaBH4 reducer and a PEG polymer. The synthesis was carried out in a threenecked flask (Figure 1) according to the reaction:

$$
2 \mathrm{FeCl} 3+6 \mathrm{NaBH} 4+18 \mathrm{H} 2 \mathrm{O} \rightarrow 2 \mathrm{Fe} 0+6 \mathrm{NaCl}+6 \mathrm{~B}(\mathrm{OH}) 3+21 \mathrm{H} 2 \text { and covered }
$$
several stages:

- dissolving $27 \mathrm{~g}$ of $\mathrm{FeCl} 3 \cdot 6 \mathrm{H} 2 \mathrm{O}$ in a mixture of $25 \mathrm{ml}$ deionized water with $75 \mathrm{ml}$ of ethyl alcohol (in a Berzelius beaker);

- addition of $0.237 \mathrm{~g}$ of PEG to the iron salt solution;

- Transferring the aqueous solution into a three-necked round-bottomed flask and magnetically shaking the solution at $900 \mathrm{rpm}$;

- Preparation of a $0.1 \mathrm{M}$ solution of sodium borohydride by dissolving NaBH4 in deionized water; the $\mathrm{NaBH} 4$ solution was introduced into a burette and dosed dropwise in the previously obtained solution;

- obtaining a colloidal solution of iron nanoparticles; chemical reduction to Fe is complete after vigorous stirring of the solution (1200 RPM) at room temperature for 15 minutes;

- Separation by centrifugation at $3000 \mathrm{rpm}$ and purification; 


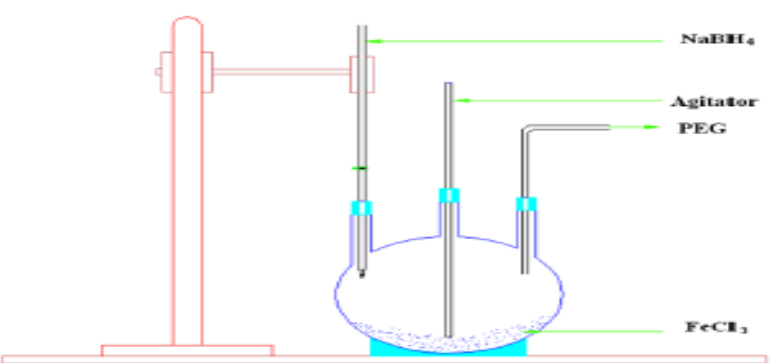

Figure 1. Installation for the synthesis of Fe metallic nanoparticles

The parameters that influenced the process were: the iron salt concentration, the nature of the solvent, the reaction time, the polymer concentration as a dispersing and anti-oxidation agent and the presence of one or two metals.

The resulting Fe nanoparticles were characterized by: Scanning Electron Microscopy (SEM), X-ray Diffraction (XRD), X-ray Dispersive Spectrofluorimetry (EDX), X-ray Fluorescence (XRF) Spectrometry, Vibrational Magnetometry ) at room temperature.

The synthesis resulted in iron particles, ranging from 29 to $108 \mathrm{~nm}$, as seen in the SEM image (figure 2) and adopts the cubic structure with centered faces of the iron crystal according to the XRD analysis (figure 3 ).

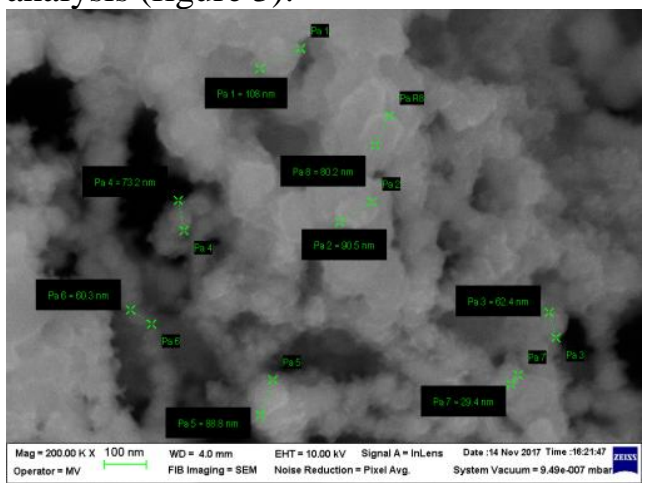

Figure 2. SEM image for Fe nanoparticles

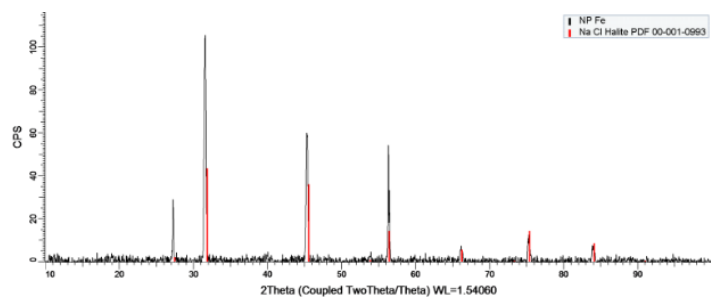

Figure 3.

$\mathrm{X}$-ray diffractogram of Fe nanoparticles obtained in the system:

$\mathrm{FeCl}_{3} \cdot 6 \mathrm{H}_{2} \mathrm{O}-\mathrm{NaBH}_{4}-\mathrm{PEG}$

Following the XRD analysis (figure 3) made on the Fe nanoparticulate sample the presence of peaks characteristic of iron and sodium chloride $\mathrm{NaCl}$ was confirmed, as confirmed by the XRF analysis (Table 1). Sodium chloride results as a by-product following iron metal salt reduction $(\mathrm{FeCl} 3.6 \mathrm{H} 2 \mathrm{O})$ and is due to insufficient sample purification.

Table 1. Results of XRF analysis for Fe nanoparticles

\begin{tabular}{|l|l|l|l|l|l|l|l|l|}
\hline Formula & Z & Concentration & Status & Line 1 & $\begin{array}{l}\text { Net } \\
\text { int. }\end{array}$ & $\begin{array}{l}\text { Calc. } \\
\text { concentration }\end{array}$ & $\begin{array}{l}\text { Stat. } \\
\text { error }\end{array}$ & LLD \\
\hline $\mathrm{Fe}$ & 26 & $47,32 \%$ & XRF 1 & $\begin{array}{l}\text { Fe KA1- } \\
\text { HR-Tr }\end{array}$ & 342,8 & 47,32 & $\begin{array}{l}0,306 \\
\%\end{array}$ & $\begin{array}{l}96,3 \\
\text { PPM }\end{array}$ \\
\hline $\mathrm{Cl}$ & 17 & $39,30 \%$ & XRF 1 & $\begin{array}{l}\text { Cl KA1- } \\
\text { HR-Tr }\end{array}$ & 59,40 & 39,3 & $\begin{array}{l}0,735 \\
\%\end{array}$ & $\begin{array}{l}84,2 \\
\text { PPM }\end{array}$ \\
\hline $\mathrm{Na}$ & 11 & $13,38 \%$ & XRF 1 & $\begin{array}{l}\text { Na KA1- } \\
\text { HR-Tr }\end{array}$ & 3,305 & 13,4 & $3,14 \%$ & $\begin{array}{l}215,0 \\
\text { PPM }\end{array}$ \\
\hline
\end{tabular}


These nanoparticles were subjected to an additional purification process and analyzed again by: SEM (Figure 4), EDX analysis (Figure 5), XRF analysis (Table 2) and VSM (Figure 7).

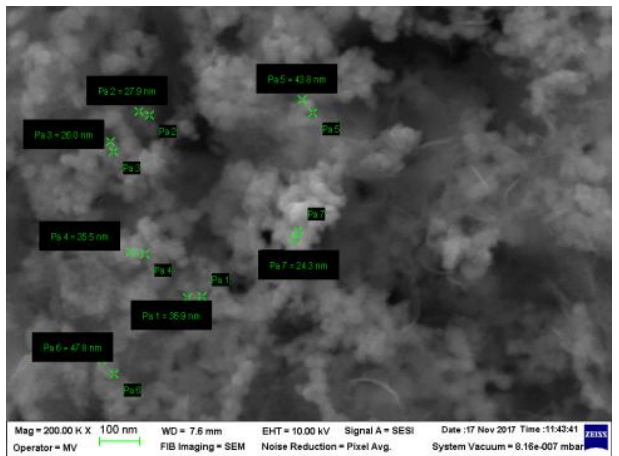

Figure 4. SEM image for additional purified Fe nanoparticles
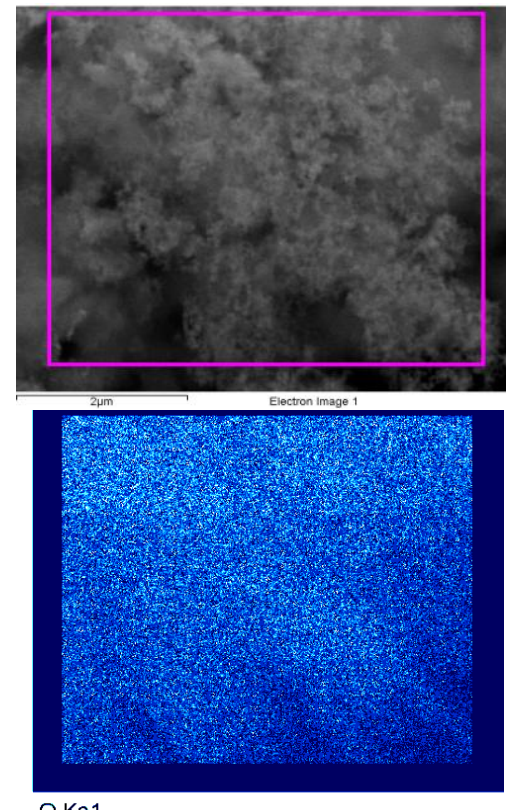

\begin{tabular}{c|cc}
\multicolumn{2}{c}{ OKa1 } & \\
\hline Element & Weight\% & Atomic\% \\
C K & 11.14 & 23.96 \\
O K & 30.34 & 48.98 \\
Fe K & 58.52 & 27.06 \\
& & \\
Totals & 100.00 & \\
\hline
\end{tabular}

Spectrum processing : No peaks omitted Processing option : All elements analyzed (Normalised) Number of iterations $=5$ Standard : C CaCO3 1-Jun-1999 12:00 AM O $\mathrm{SiO} 2$ 15-Jul-2010 06:48 PM $\mathrm{Fe} \quad \mathrm{Fe}$ 1-Jun-1999 12:00 AM

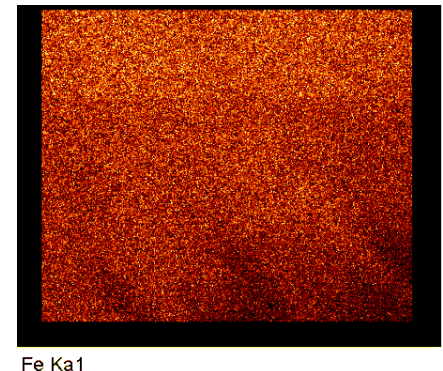

Fe Ka1

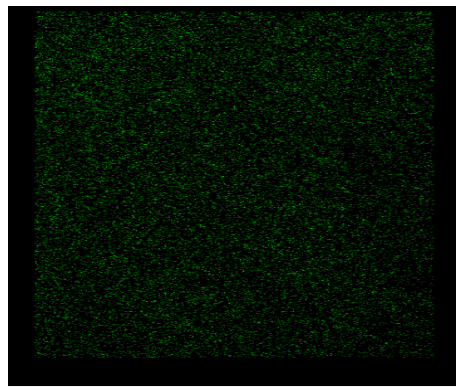

$\mathrm{CKa} 12$

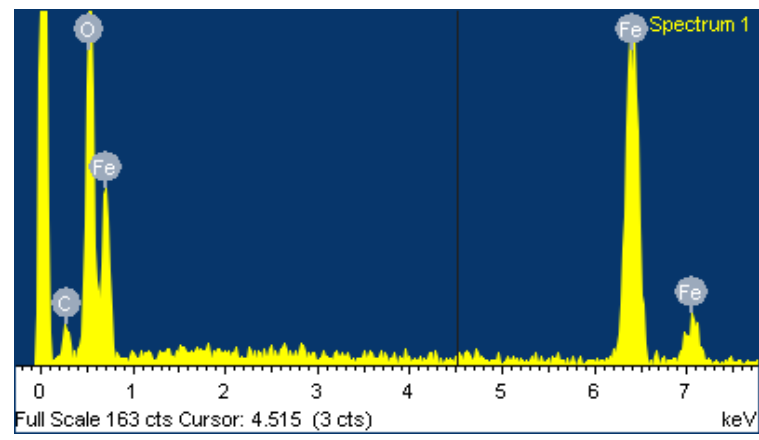

Figure 5. EDX spectrum for additional purified $\mathrm{Fe}$ nanoparticles

Table 2. Results of XRF analysis for Fe nanoparticles after advanced purification 
Volume 1, Issue 1, 2019

ISSN: 2668-0416

Thoth Publishing House

\begin{tabular}{|l|l|l|l|l|l|l|l|l|}
\hline Formula & $\mathrm{Z}$ & Concentration & Status & Line 1 & $\begin{array}{l}\text { Net } \\
\text { int. }\end{array}$ & Stat. error & LLD & $\begin{array}{l}\text { Analyzed } \\
\text { layer }\end{array}$ \\
\hline $\mathrm{Fe}$ & 26 & $97,38 \%$ & XRF 1 & $\begin{array}{l}\text { Fe KA1-HR- } \\
\mathrm{Tr}\end{array}$ & 467,5 & $0,262 \%$ & $\begin{array}{l}93,8 \\
\text { PPM }\end{array}$ & $30,0 \mathrm{um}$ \\
\hline $\mathrm{Cl}$ & 17 & $1,57 \%$ & XRF 1 & $\begin{array}{l}\text { Cl KA1-HR- } \\
\mathrm{Tr}\end{array}$ & 1,471 & $4,95 \%$ & $\begin{array}{l}81,2 \\
\text { PPM }\end{array}$ & $2,72 \mathrm{um}$ \\
$\mathrm{Na}$ & 11 & $0,88 \%$ & XRF 1 & $\begin{array}{l}\text { Na KA1-HR- } \\
\mathrm{Tr}\end{array}$ & 0,0853 & $19,4 \%$ & & $0,263 \mathrm{um}$ \\
\hline
\end{tabular}

The XRF analysis revealed an increase in the Fe content in the sample from $47.32 \%$ to $97.38 \%$. Content in the $\mathrm{Cl}$ and $\mathrm{Na}$ elements has fallen. Concentration in $\mathrm{NaCl}$ diminished. Figures 6 and 7 show the hysteresis curves of $\mathrm{Fe}$ nanoparticles before and after purification. The purified sample has a ferromagnetic behavior at room temperature: MS of $14.38 \mathrm{emu} / \mathrm{g}, \mathrm{Mr}$ of $4.14 \mathrm{emu} / \mathrm{g}$ and $\mathrm{Hc}$ coercivity of 387.2 Oe. After purification, we have an improvement in the magnetic behavior of the $\mathrm{Fe}$ nanoparticle sample (Table 3 ).

Table 3. VSM measurements for Fe nanoparticles before and after purification

\begin{tabular}{|c|l|c|c|c|}
\hline Nr. & \multicolumn{1}{|c|}{ Sample code } & $\begin{array}{c}\mathrm{M}_{\mathrm{r}} \\
(\mathrm{emu} / \mathrm{g})\end{array}$ & $\begin{array}{c}\mathrm{M}_{\mathrm{S}} \\
(\mathrm{emu} / \mathrm{g})\end{array}$ & $\begin{array}{c}\mathrm{H}_{\mathrm{c}} \\
(\mathrm{Oe})\end{array}$ \\
\hline 1 & Fe Nanoparticles & 2.68 & 9.39 & 353.2 \\
\hline 2 & $\begin{array}{l}\text { Fe nanoparticles after advanced } \\
\text { purification }\end{array}$ & 4.14 & 14.38 & 387.2 \\
\hline
\end{tabular}

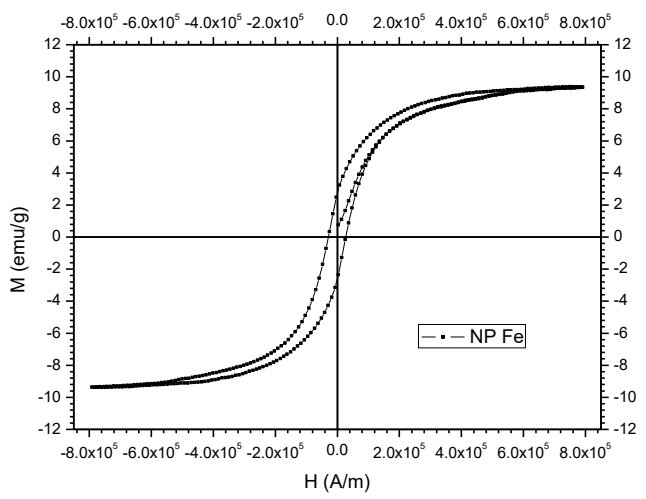

Figure 6. $\mathrm{H}$-function curve of $\mathrm{H}$ for $\mathrm{Fe}$ nanoparticles impregnated with $\mathrm{NaCl}$.

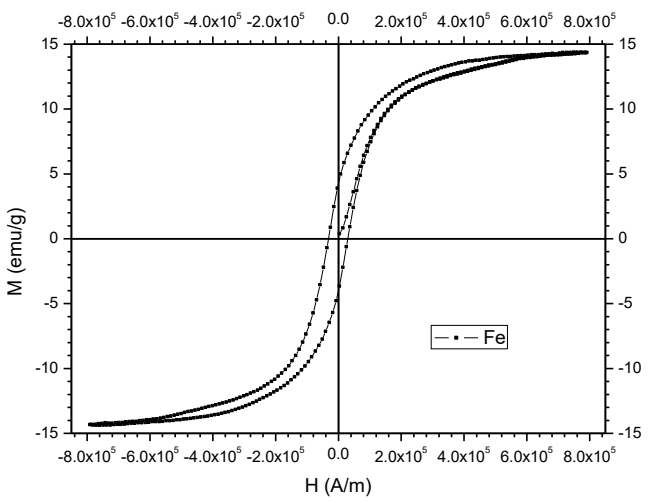

Figure 7 .The $\mathrm{H}$-function of $\mathrm{H}$ for $\mathrm{Fe}$ nanoparticles after advanced purification.

From the analysis of magnetic results, the increase in specific saturation magnetisation for advanced purified Fe nanoparticles was observed, from $9.39 \mathrm{emu} / \mathrm{g}$ to $14.38 \mathrm{emu} / \mathrm{g}$. Also, there is an increase in the coercive field value, which can probably be explained by the increase in magnetic interaction forces as a result of particle size reduction $(24-47 \mathrm{~nm})$, as seen from the SEM image (Figure 4)

The iron-cobalt alloy nanoparticles were obtained in the system: $\mathrm{FeCl} 3.6 \mathrm{H} 2 \mathrm{O}$ - $\mathrm{Co}(\mathrm{NO} 3) 2.6 \mathrm{H} 2 \mathrm{O}$ - NaBH4 - PEG

The iron-cobalt alloy nanoparticles were prepared by the same method as iron nanoparticles (the metal salt reduction method), $\mathrm{FeCl} 3.6 \mathrm{H} 2 \mathrm{O}$ and $\mathrm{Co}$ (NO3) 2.6H2O.

The resulting nanoparticles were characterized by: SEM, X-ray diffraction (XRD), X-ray dispersive spectrofluorimetry (EDX), X-ray fluorescence (XRF) spectrometry, Vibration-proof magnetometry room's temperature 
To obtain the cobalt-iron alloy, the Fe-Co molar ratio was 1: 1, confirmed by the EDX and XRF analysis. The SEM image highlights the formation of spherical nanoparticles. Particle sizes ranging from 25-83 nm (SEM image - Figure 8) were obtained.

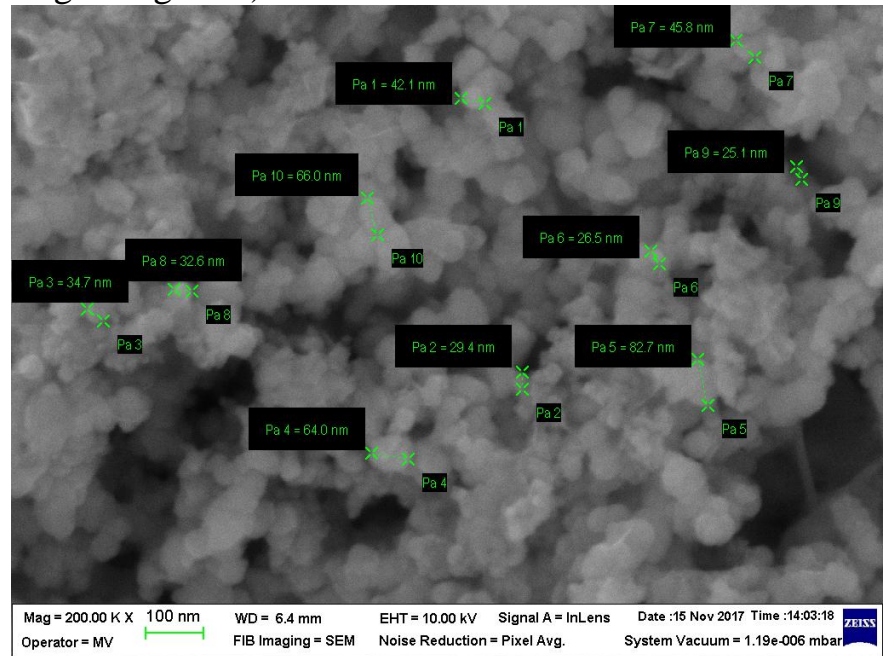

Figure 8. SEM image for Fe-Co alloy nanoparticles

Figure 9 shows the X-ray diffractogram of the Fe-Co alloy particles obtained in the $\mathrm{FeCl} 3.6 \mathrm{H} 2 \mathrm{O}$ Co (NO3) 2.6H2O-NaBH4-PEG system. The presence of peaks characteristic of iron and cobalt was found; the presence of the elements is also confirmed by EDX (Figure 10) and XRF analysis (Table 4).

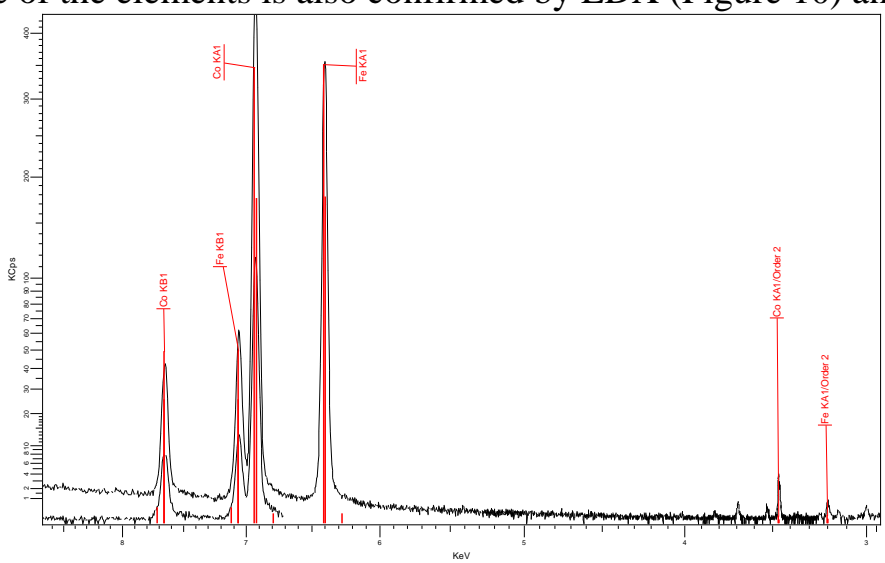

Figure 9. X-ray diffractogram of obtained Fe-Co alloys nanoparticles:

$\mathrm{FeCl} 3.6 \mathrm{H} 2 \mathrm{O}$ - $\mathrm{Co}(\mathrm{NO} 3)$ 2.6H2O - NaBH4-PEG

Table 4 Results of XRF analysis for Fe-Co nanoparticles

\begin{tabular}{|l|l|l|l|l|l|l|l|l|}
\hline Formula & Z & Concentration & Status & Line 1 & $\begin{array}{l}\text { Net } \\
\text { int. }\end{array}$ & Stat. error & LLD & $\begin{array}{l}\text { Analyzed } \\
\text { layer }\end{array}$ \\
\hline Co & 27 & $50,51 \%$ & XRF 1 & Co KA1-HR-Tr & 478,8 & $0,259 \%$ & 186,0 PPM & 33 um \\
\hline Fe & 26 & $49,49 \%$ & XRF 1 & Fe KA1-HR-Tr & 358,2 & $0,300 \%$ & 100,4 PPM & 26,8 um \\
\hline
\end{tabular}

Figure 10 shows the EDX spectrum for Co-Fe nanoparticles 

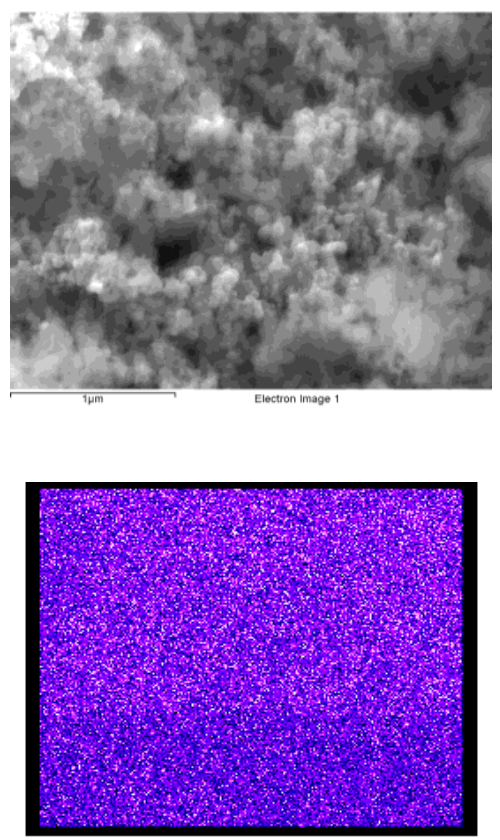

Co Ka1
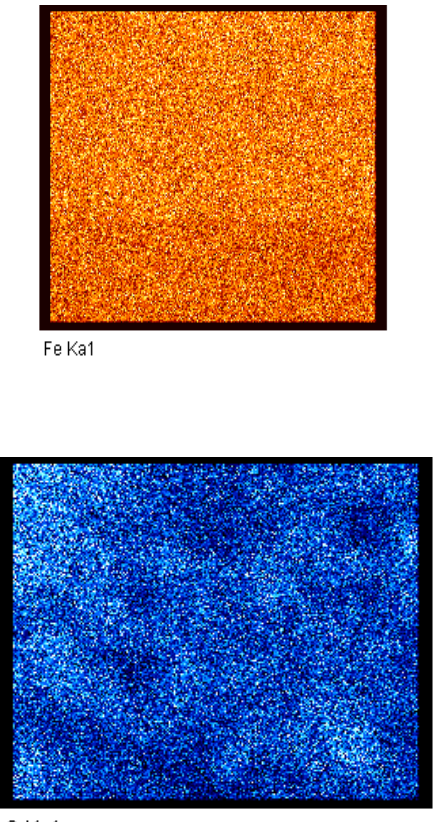

o Ka1

\begin{tabular}{c|cc}
\hline Element & Weight\% & Atomic\% \\
O K & 12.97 & 34.84 \\
Fe K & 41.76 & 32.14 \\
Co K & 45.28 & 33.02 \\
Totals & 100.00 & \\
\hline
\end{tabular}

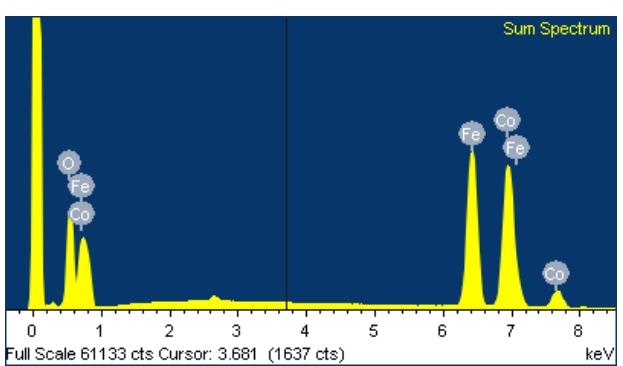

Figure 10. EDX spectrum for Fe-Co nanoparticles

Magnetic measurements on the Fe-Co alloy nanoparticles obtained were performed at room temperature. Figure 11 shows the hysteresis curves of Fe-Co alloy nanoparticles. The sample of $\mathrm{Fe}-\mathrm{Co}$ alloy nanoparticles has a ferromagnetic behavior at room temperature: MS of $44.26 \mathrm{emu} / \mathrm{g}$, Mr of $16.26 \mathrm{emu} / \mathrm{g}$, and Hc coercivity of 436.3 Oe (Table 5).

Table 5. VSM measurements for Fe-Co alloy nanoparticles

\begin{tabular}{|c|c|c|c|c|}
\hline No & Sample code & $\mathrm{M}_{\mathrm{r}}(\mathrm{emu} / \mathrm{g})$ & $\mathrm{M}_{\mathrm{S}}(\mathrm{emu} / \mathrm{g})$ & $\mathrm{H}_{\mathrm{c}}(\mathrm{Oe})$ \\
\hline 1 & Nanoparticles Fe-Co alloy & 16.26 & 44.26 & 436.3 \\
\hline
\end{tabular}

$\mathrm{Fe}$ and $\mathrm{Fe}-\mathrm{Co}$ nanoparticle samples were synthesized as compared to a metallurgical micronized a $\mathrm{Fe}$ Fe powder at Hoeganaes Europe SA Buzau.

The a Fe Fe particles (originated from Hoeganaes Europe SA Buzau) were characterized by: SEM electronic scanning microscopy, X-ray diffraction (XRD), X-ray dispersion spectrofluorometry (EDX), X-ray fluorescence spectroscopy (XRF) vibration sample (VSM) at room temperature.A Fe Fe 
particles were characterized by SEM electronic scanning microscopy (Figure 12). This image shows particle sizes ranging from $21-40 \mu \mathrm{m}$.

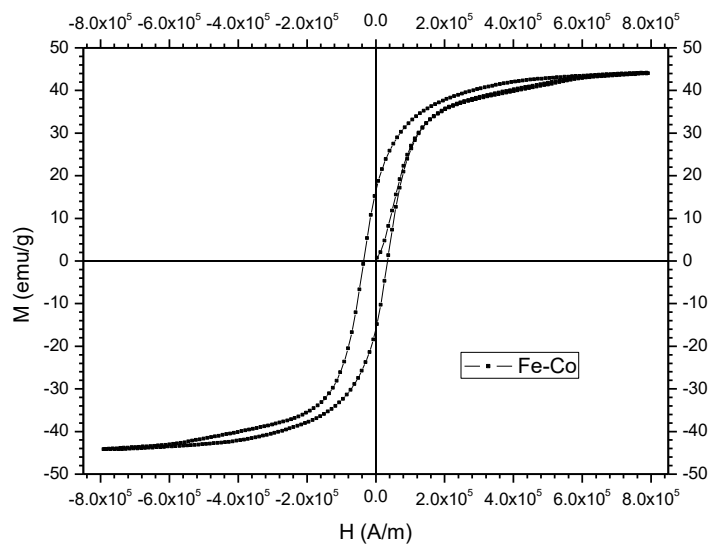

Figure 11. $\mathrm{H}$-function curve of $\mathrm{H}$ for $\mathrm{Fe}-\mathrm{Co}$ alloy nanoparticles

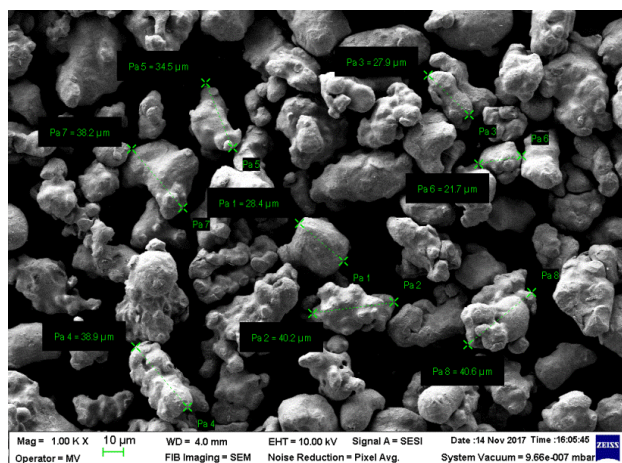

Figure 12. SEM image for $\alpha \mathrm{Fe} F$ pe particles

Figure 13 shows the X-ray diffractogram of $\alpha$ Fe particles.

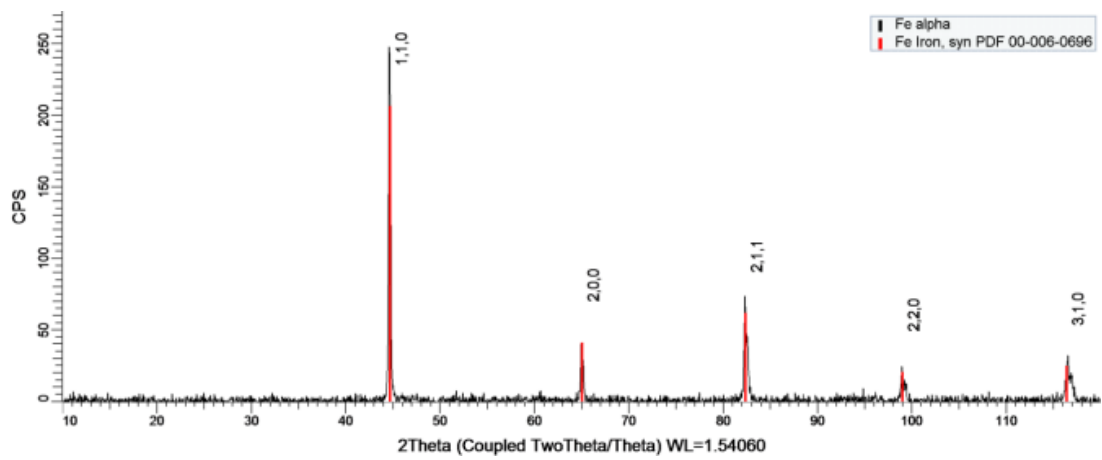

Figure 13. X-ray diffractogram of $\alpha$ Fe particles

The presence of iron peaks was found, fact confirmed by EDX analysis (Figure 14) and XRF (Table 6).

The crystallite size is $\mathrm{D}=37 \mathrm{~nm}$ and the elemental cell parameter $\mathrm{a}=2.8696 \mathrm{~A}$. 


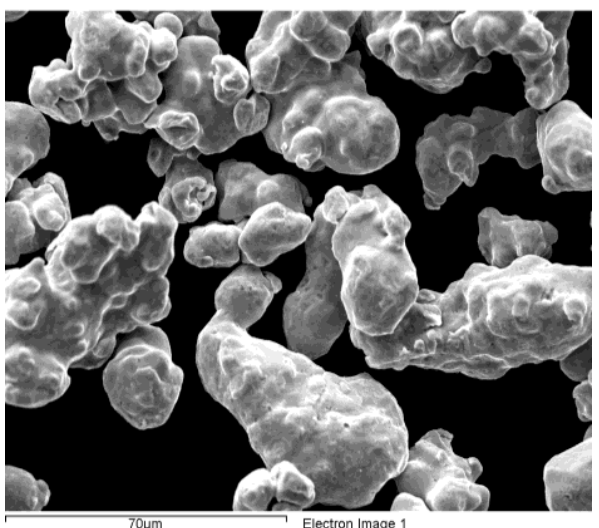

Comment: Alfa Fe
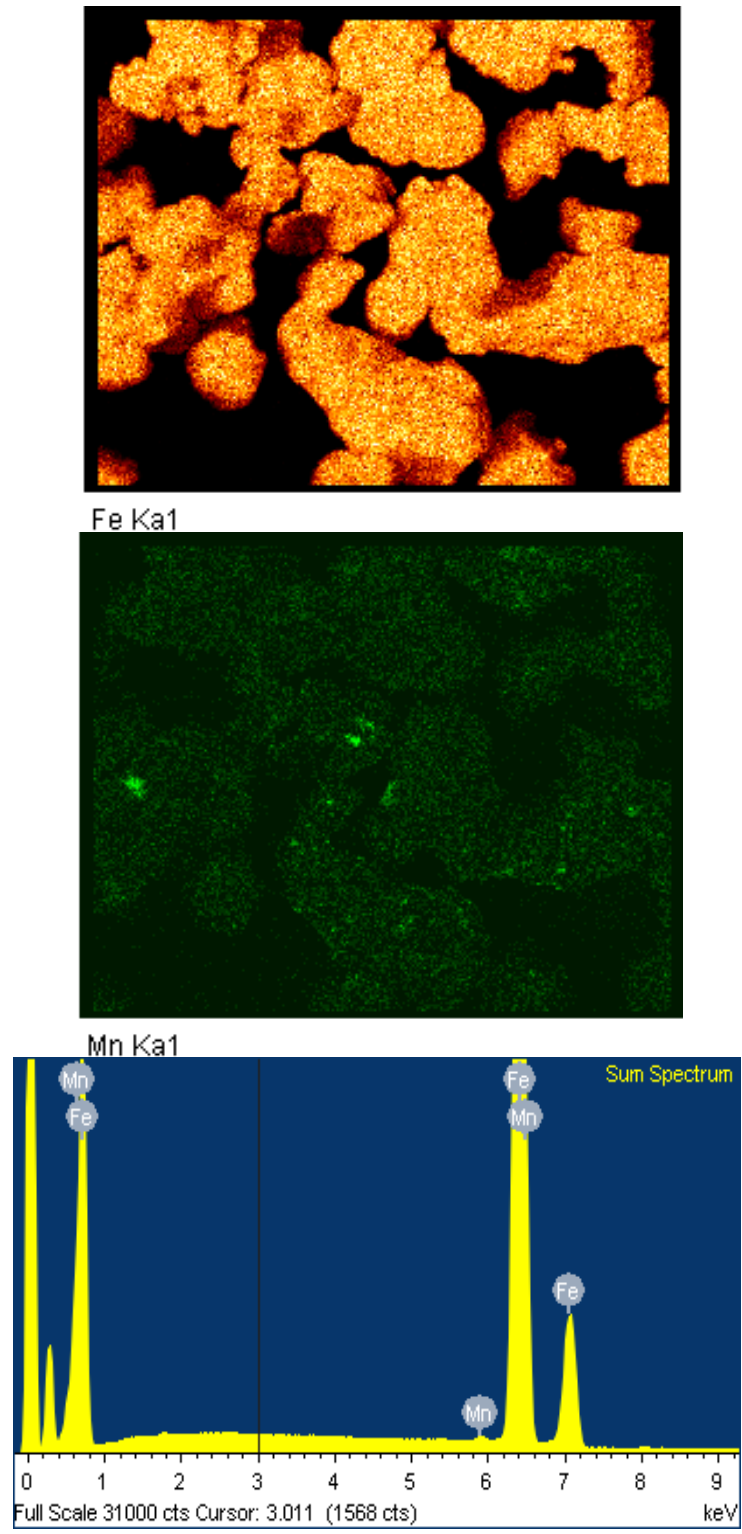

Spectrum processing :

Peaks possibly omitted : $1.763,7.465,8.031$

$\mathrm{keV}$;Processing option : All elements

analyzed (Normalised);

Number of iterations $=1$

Standard :

Mn Mn 1-Jun-1999 12:00 AM

Fe Fe 1-Jun-1999 12:00 AM

\begin{tabular}{l|ll}
\hline Element & Weight\% & Atomic\% \\
Mn K & 0.49 & 0.50 \\
Fe K & 99.51 & 99.50 \\
Totals & 100.00 & \\
\hline
\end{tabular}

Figure 14. EDX spectrum for $\alpha$ Fe Fe particles

Table 6. XRF for $\alpha$ Fe particles

\begin{tabular}{|l|l|l|l|l|}
\hline Formula & $\mathrm{Z}$ & Concentration & Line 1 & $\begin{array}{l}\text { Analyzed } \\
\text { layer }\end{array}$ \\
\hline $\mathrm{Fe}$ & 26 & $99,62 \%$ & Fe KA1-HR-Tr & $30,0 \mathrm{um}$ \\
\hline $\mathrm{Mn}$ & 25 & $0,18 \%$ & Mn KA1-HR-Tr & $23,9 \mathrm{um}$ \\
\hline $\mathrm{Cu}$ & 29 & $0,17 \%$ & Cu KA1-HR-Tr & $6,9 \mathrm{um}$ \\
\hline $\mathrm{Cr}$ & 24 & $0,03 \%$ & Cr KA1-HR-Tr & $18,8 \mathrm{um}$ \\
\hline
\end{tabular}

Magnetic measurements on the obtained $\alpha$-Fe particles were performed at room temperature.

Figure 15 shows the hysteresis curves of the $\alpha$ Fe particles. The sample has a ferromagnetic behavior 
at room temperature and shows: MS of $255.87 \mu \mathrm{g} / \mathrm{g}$, Mr of $17.79 \mathrm{emu} / \mathrm{g}$ and Hc coercivity of $281.1 \mathrm{Oe}$ (Table 7).

Table 7.

\begin{tabular}{|c|c|c|c|c|}
\hline Nr. & $\begin{array}{c}\text { Sample } \\
\text { code }\end{array}$ & $\begin{array}{c}\mathrm{M}_{\mathrm{r}} \\
(\mathrm{emu} / \mathrm{g})\end{array}$ & $\begin{array}{c}\mathrm{M}_{\mathrm{S}} \\
(\mathrm{emu} / \mathrm{g})\end{array}$ & $\begin{array}{c}\mathrm{H}_{\mathrm{c}} \\
(\mathrm{Oe})\end{array}$ \\
\hline 3 & $\alpha \mathrm{Fe}$ & 17.79 & 255.87 & 281.1 \\
\hline
\end{tabular}

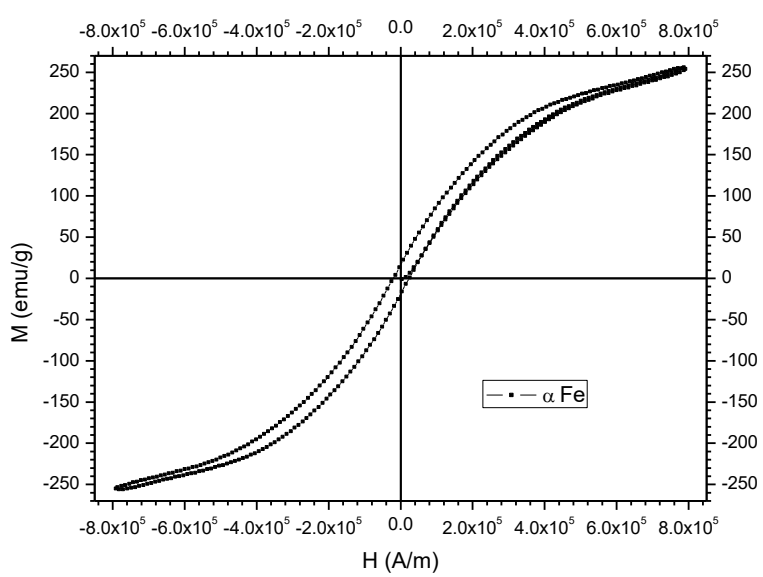

Figure 15. $\mathrm{H}$-function curve $\mathrm{H}$ for $\alpha \mathrm{Fe}$ particles

\section{Methods used to obtain composite materials with fiber inclusions}

The methods comprise processes for indirect or direct incorporation of the fibers. Indirect incorporation involves separate fabrication of fibers, followed by their addition by soldering, infiltration, electrolytic deposition of the matrix around the fibers, chemical deposition, or plastic deformation of the matrix (hot pressing of alternating fibers and matrices).

Direct incorporation takes into account fiber formation and matrix growth simultaneously, either by solidification or by cold plastic deformation of the entire structure [29].

The spray method is used for the manufacture of composite materials using an open die - see Figure 16. The matrix and inclusions (small cut fibers) are sprayed into a reusable mold, being allowed to dry at room temperature. Polymer matrices and glass fibers are generally used.

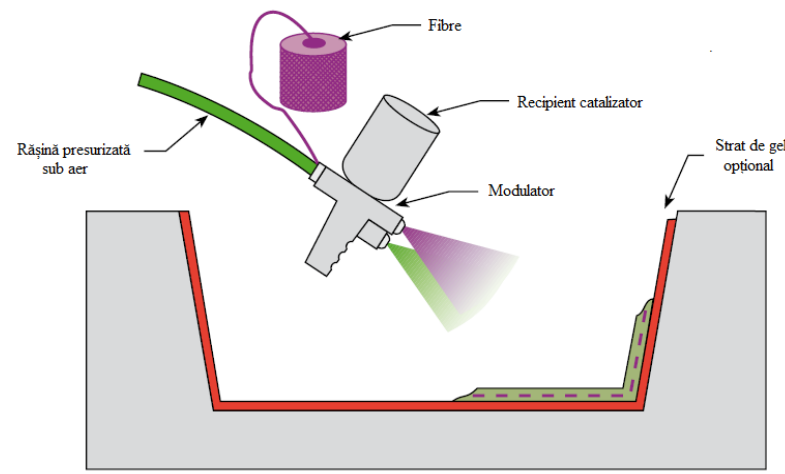

Figure 16. Spray method (adaptation after [30])

The method of transferring the matrix into the mold (Figure 17) involves adding the matrix to a mold in which the fibers are already present. There is a pressing process that leads to the desired composite material. Drying can be done either at room temperature or at elevated temperatures. 


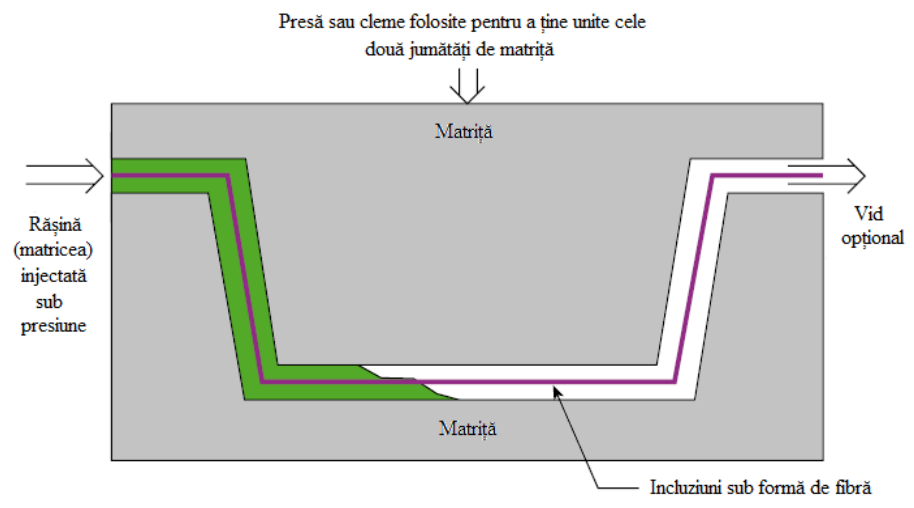

Figure 17. Matrix in mold transfer method [30]

The pultrusion method involves a process of making fiber composites of the same section. The fibers are pulled through the liquid matrix, then into a heated mold. This mold finishes the process of impregnating the fibers and dries the material giving it its final shape. All types of polymeric matrix can be used for this method. The method has the principle shown - Fig. 8

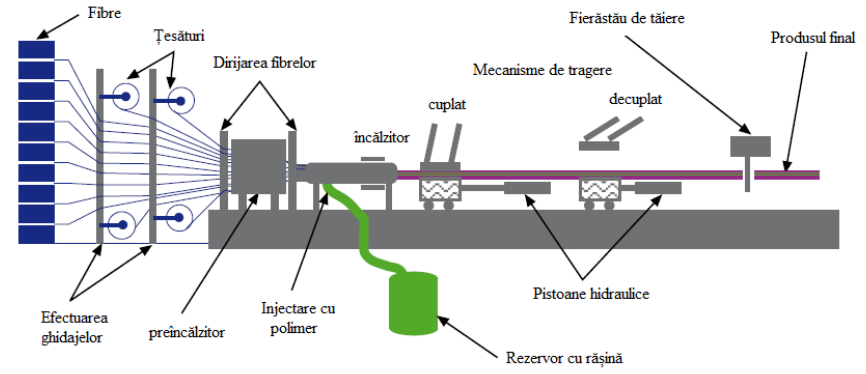

Figure 18. The pultrusion method [30]

The manual arranging method involves placing the fibers with your hand in the mold (Figure 19). Using brushes or rollers, the matrix is impregnated with fibers [30]. In this case drying is carried out at room temperature.

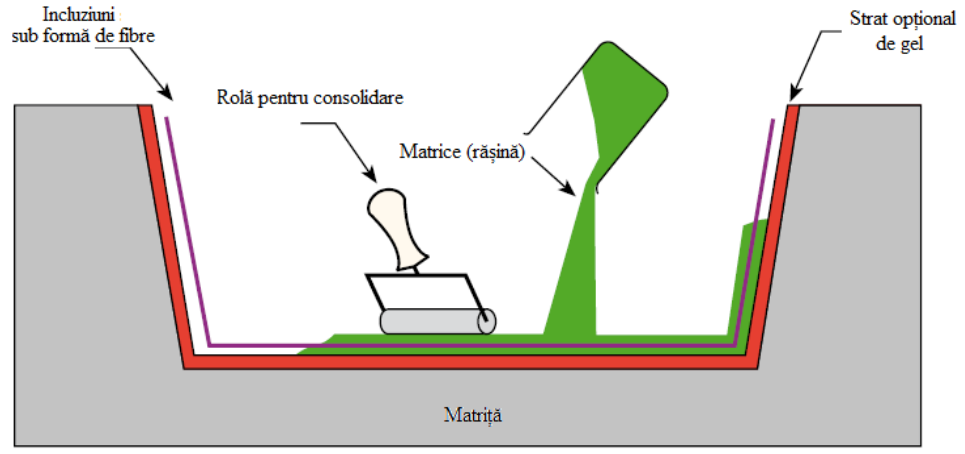

Figure 19. Method of impregnating the matrix with fibers using rollers [30]

A recent method of obtaining fibrous fiber composites is presented in [31] by An et al. They propose a method of obtaining composite materials with carbon fiber inclusions based on a chemical method. Embedding is done using chemical reactions - aerosol-assisted vapor deposition. 


\section{Methods used to obtain magnetic composite materials with particle inclusions}

The simplest way to obtain composite materials with particle inclusions is by mechanically mixing the powder with polymers and curing agents [32]. After the mixing is carried out, the material obtained is subjected to a drying, heating, sintering process, etc. The manufacturing process is very simple: the powder is isolated using an inorganic layer. Using different metallurgical processes, the material is poured into a mold and compacted, subjected to high pressures. After the compaction process is completed, the material is removed from the mold. Often, a lubricant is added to the initial mixture to facilitate the pressing and extraction process of the press material [33].

Another method used to produce soft composite materials is to press inclusions (generally pure $\mathrm{Fe}$ ) using high pressure [34]. The iron powder is processed so that each grain of material has a magnetic insulating layer (polymeric matrix). The purpose of this layer is to magnetically isolate the grains in order for the material to become more efficient and to limit the loss by eddy currents. Particles are obtained, coated with an insulating layer, between 2 and $200 \mu \mathrm{m}$. The powder is pressed into closed molds and a semifinished material is obtained. The curing process follows in controlled atmosphere and generally at temperatures below $700{ }^{\circ} \mathrm{C}$. Additives and adhesives can be added to provide increased resistance to the material. Often, a lubricant is added to the initial mixture to facilitate the pressing and extraction process of the press material

Multiple methods can be used to obtain composite materials with particle inclusions. One of them is presented in [35]. In this paper the authors present several methods of obtaining magnetic nanocomposite materials. Co-based composites can be sintered using the thermal decomposition method in the presence of surfactants (chemicals that can reduce surface tension - an example is oleic acid $\mathrm{C} 18 \mathrm{H} 34 \mathrm{O} 2$ ). Each inclusion is covered with these agents to prevent their agglomeration, resulting in a magnetic nanocomposite material with cubic symmetry.

Another method of obtaining, all chemical - non-homogeneous nucleation in an organic solvent, for the production of nanoparticles used in composite materials [35]. This method involves increasing the material to obtain core-type core materials.

Composite materials are also used successfully in the electronics industry. Using this type of material, the electronic components can be miniaturized so as to occupy less space on the printed circuit boards [36].

\section{Conclusions}

A composite material can be described as a combination of two or more distinct materials that at microscopic level maintain their own properties and structure, but at macroscopic level the resulting material appears homogeneous. The entire assembly has characteristics and properties different from each component material. Because there are many ways to get composite materials, it's important to study their structure and properties

\section{References}

[1] Mehdi Zamanpour, Cobalt-based Magnetic Nanoparticles: Design, Synthesis and Characterization, Dissertation Northeastern University, Bosto, 2014

[2] Willard M. A., L. K. Kurihara, E. E. Carpenter, S. Calvin and V. G. Harris, Chemically prepared magnetic nanoparticles, International materials reviews, 49, pp. 125- 170, 2004

[3] Shi Y., J. Ding, X. Liu, J. Wang, NiFe2O4 ultrafine particles prepared by coprecipitation/mechanical alloying, Magnetism and Magnetic Materials, 205, pp. 249-254, 1999

[4] Zhiqiang J., W. Tang, J. Zhang, H. Lin, Y. Du., Magnetic properties of isotropic SrFe12O19 fine particles prepared by mechanical alloying, Magnetism and Magnetic Materials, 182, pp. 231-237, 1998

[5] Chen J. and D. E. Nikles, Preparation of acicular $\alpha$-Fe nanoparticles in a lamellar liquid crystalline 
phase, IEEE Transactions on Magnetics, 32, pp. 4478-4480, 1996

[6] Chen M., B. Tang and D. E. Nikles, Preparation of iron nanoparticles by reduction of acicular $\beta$ FeOOH particles, IEEE Transactions on Magnetics, 34, pp. 1141-1176, 1998

[7] Krehula S., S. Popovic and S. Music, Synthesis of acicular $\alpha$-FeOOH particles at a very high $\mathrm{pH}$, Materials Letter, 54, pp. 108-113, 2002

[8] Chakroune N., G. Viau, C. Ricolleau, F. Fievet-Vincent and F. Fievet, Cobalt based anisotropic particles prepared by the polyol process, Materials Chemistry, 13, pp. 312-318, 2003

[9] Turkevich J., P. C. Stevenson, J. Hillier, A study of the nucleation and growth processes in the synthesis of colloidal gold, Discussions of the Faraday Society, 11, pp. 55-57, 1951

[10] Turkevich J., G. Kim, Palladium: preparation and catalytic properties of particles of uniform size, Science 169, pp.873-875, 1970

[11] Turkevich J., Nucleation and Growth Process in the Synthesis of Colloidal Gold, Gold Bulletin, 18, pp. 86-88, 1985

[12] Peng X., L. Manna, W. Yang, J. Wickham, E. Scher, A. Kadavanich and A. P. Alivisatos, Shape control of CdSe nanocrystals, Nature, 404, pp. 59-61, 2000

[13] Murray B. C., C. R. Kagan, M. G. Bawendi, Synthesis and characterization of monodisperse nanocrystals and close-packed nanocrystals assemblies, Annual Review of Materials Science, 30, pp. 545-610, 2000

[14] LaMer V. K. and M.D. Barnes, Theory, Production and mechanism of formation of monodispersed hydrosols, Journal of Colloidal Science, 1, pp. 71-77, 1946

[15] Lalena, J. N., D. A. Cleary, E. E. Carpenter, N. F. Dean, Inorganic Materials Synthesis and Fabrication; John Wiley \& Sons: New Jersey, pp. 41-70, 2008

[16] Nielsen A. E., Kinetics of precipitation, Pergamon Press, New York, pp. 13-20, 1964

[17] Walton A. G., The formation and properties of precipitates, Robert Krieger, New York, pp. 5-54, 1979

[18] Rosen M. J. and Kunjappu J. T, Interfacial Phenomena, John Wiley \& Sons, New Jersey, pp. 198, 2010

[19] H. Bonnemann, W. Brijoux, R. Brinkmann, N. Matoussevitch, N. Waldofner, N. Palina, H. Modrow, A size-selective synthesis of air stable colloidal magnetic cobalt nanoparticles, Inorganica Chimica Acta 350, pp. 617-624, 2003

[20] I. Lisiecki, M. P. Pileni, Synthesis of Well-Defined and Low Size Distribution Cobalt Nanocrystals: The Limited Influence of Reverse Micelles, Langmuir, 19 (22), pp 9486-9489, 2003

[21] B. Chaudret, K. Philippot, Organometallic Nanoparticles of Metals or Metal Oxides, Oil \& Gas Science and Technology-Rev. IFP, Vol.62, No.6, pp. 799-817, 2007

[22] Gangopadhyay S, Yaang Y, Hadjipanayis G.C. et al, Magnetic and structural properties of vapordeposited Fe-Co alloy particles, J.Appl.Phis., 76 10, pp. 6319-6321, 1994

[23] Chen J.P., Sorensen C.M., Klabunde K.J., Hadjipanayis G.C., Magnetic properties of nanophase cobalt particles synthesised in inversed micelles, J.Appl.Phis., 76, pp. 6316-6318, 1994 .

[24] Poul L., Ammar S., Jouini N., F. Fievet, F. Villain, Synthesis Synthesis of Inorganic Compounds (Metal, Oxide and Hydroxide) in Polyol Medium: A Versatile Route Related to the Sol-Gel Process, Journal of Sol-Gel Science and Technology, Volume 26, Issue 1-3, pp 261-265, 2003

[25] Jean-Marie Berthelot, Composite materials: mechanical behavior and structural analysis, Springer, New York, 1999

[26] D. Gay, Matériaux composites, Hermes, Paris, 1997

[27] H. Altenbach, J. Altenbach, W. Kissing, Mechanics of composite structural elements, SpringerVerlag Berlin Heidelberg, Germania, 2004

[28] J.Weiss, C. Bord, Les matériaux composites - Structure, Constituants, Fabrication, 1983, Paris , l'usine nouvelle.

[29] V. Suciu, M. V. Suciu, Studiul materialelor, Editura Fair Partners, București, 2008 
[30] F.L. Matthews, R.D. Rawlings, Composite materials: Engineering and Science, Woodhead Publishing Limited, England, 1999.

[31] Feng An, Chunxiang Lu, Yonghong Li, Jinhai Guo, Xiaoxuan Lu, Huibin Lu, Shuqing He, Yu Yang, Preparation and characterization of carbon nanotube-hybridized carbon fiberto reinforce epoxy composite, Materials and Design, vol. 33, 2012, pp.197-202

[32] F.L. Matthews, R.D. Rawlings, Composite materials: Engineering and Science, Woodhead Publishing Limited, England, 1999.

[33] B. Sareni, L. Krahenbuhl, A. Beroual, C. Brosseau, Effective dielectric constant of random composite materials, Journal of Applied Physics, vol. 81, nr. 5, 1997

[34] A. Bordianu, O. de la Barrière, O. Bottauscio, M. Chiampi, A. Manzin, A multiscale approach to predict classical losses in soft magnetic composites, IEEE Transactions on Magnetics, vol. 48, nr. 4, 2012, pp. 1537-1540

[35] T. Wen, K.M. Krishnan, Cobalt-based magnetic nanocomposites: fabrication, fundamentals and applications, Journal of Physics D: Applied Physics, vol. 44, 2011

[36] Hua Su, Huaiwu Zhang, Xiaoli Tang, Yulan Jing, Zhiyong Zhong, Dielectric and magnetic properties of low-temperature fired NiCuZn-BaTiO3 composites, Journal of Magnetism and Magnetic Materials, vol. 321, 2009, pp. 2763-2766 